\title{
Off-axis effects on the multipulse structure of sperm whale usual clicks with implications for sound production
}

\author{
Walter M. X. Zimmer ${ }^{\text {a) }}$ \\ NATO Undersea Research Centre, V.le San Bartolomeo 400, I-19138 La Spezia, Italy \\ Peter T. Madsen \\ Woods Hole Oceanographic Institution, Woods Hole, Massachusetts 02543 \\ Valeria Teloni \\ Department of Zoophysiology, Biological Institute, University of Aarhus, DK-8000 Aarhus C, Denmark
}

Mark P. Johnson and Peter L. Tyack

Woods Hole Oceanographic Institution, Woods Hole, Massachusetts 02543

(Received 12 May 2005; revised 26 August 2005; accepted 31 August 2005)

\begin{abstract}
Sperm whales (Physeter macrocephalus) produce multipulsed clicks with their hypertrophied nasal complex. The currently accepted view of the sound generation process is based on the click structure measured directly in front of, or behind, the whale where regular interpulse intervals (IPIs) are found between successive pulses in the click. Most sperm whales, however, are recorded with the whale in an unknown orientation with respect to the hydrophone where the multipulse structure and the IPI do not conform to a regular pulse pattern. By combining far-field recordings of usual clicks with acoustic and orientation information measured by a tag on the clicking whale, we analyzed clicks from known aspects to the whale. We show that a geometric model based on the bent horn theory for sound production can explain the varying off-axis multipulse structure. Some of the sound energy that is reflected off the frontal sac radiates directly into the water creating an intermediate pulse $\mathrm{p} 1 / 2$ seen in off-axis recordings. The powerful $\mathrm{p} 1$ sonar pulse exits the front of the junk as predicted by the bent-horn model, showing that the junk of the sperm whale nasal complex is both anatomically and functionally homologous to the melon of smaller toothed whales. (C) 2005 Acoustical Society of America. [DOI: 10.1121/1.2082707]
\end{abstract}

PACS number(s): 43.80.Ka [WWA]

Pages: $3337-3345$

\section{INTRODUCTION}

Based on the observation that sperm whales produce multipulsed clicks (Backus and Schevill, 1966), Norris and Harvey (1972) advanced the idea that the hypertrophied nasal complex of the sperm whale operates as a sound generator. They suggested that a single initial sound pulse is generated at the phonic lips (Fig. 1) and then reflected from air sacs at the anterior and posterior ends of the large spermaceti compartments of the nose to produce a multipulsed click. They conjectured that the primary sound pulse of highest amplitude is projected directly into the water and that the following pulses of decaying amplitude are successively delayed by the two-way travel time between the air sacs of the nose.

Møhl (1978; 2001) amended the Norris and Harvey theory by proposing that the bulk of the sound energy is directed backwards into the spermaceti organ and that only a small fraction of energy leaks directly into the water in the creation of a weak 0 pulse. Most of the sound energy travels through the spermaceti organ and the junk complex before emission into the water as a narrowly focused p1 pulse (Møhl et al., 2003; Cranford, 1999). Recent findings have corroborated the basics of this so-called bent horn model

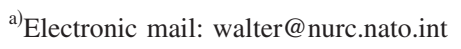

(Møhl et al., 2003) by showing that sound is indeed generated in the nose (Madsen et al., 2003) and that excitement of the system with a pressure transient at the phonic lips (Fig. 1) produces multiple pulses with an interpulse interval (IPI) that matches the two-way travel time back and forth between the air sacs (Møhl, 2001; Møhl et al., 2003).

Møhl et al. (2000; 2003) have demonstrated that the p1 pulse is highly directional with some on-axis source levels in excess of $240 \mathrm{~dB}_{\mathrm{pp}}$ re: $1 \mu \mathrm{Pa}$, which supports both the sonar function of these clicks and the bent horn amendments to the Norris and Harvey theory. Zimmer et al. (2005) corroborated these findings with a different experimental approach and found, in consistency with the bent-horn model, that the weaker p0 pulse has a broad backward-directed beam whereas the highly-directional p1 pulse is projected forward from the whale (Zimmer et al., 2005).

It follows from both the original Norris and Harvey theory and the bent horn model that the IPIs between outgoing pulses should be related to the distance between the reflective air sacs. Since there is an allometric relationship between the size of the nose and the overall body size of the whale (Nishiwaki et al., 1963), it can be inferred that the whales may convey size information in every click they make to conspecifics and interested bioacousticians alike (Norris and Harvey, 1972). 

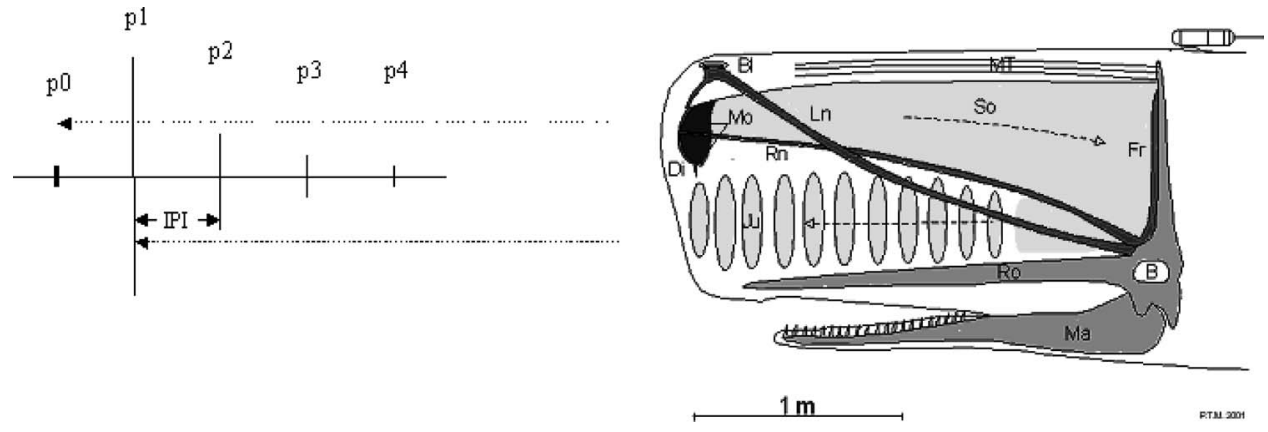

FIG. 1. Bent-horn model of sperm whale sound generation [modified from Fig. 1 of Madsen et al. (2002a). B, brain; Bl, blow hole; Di, distal air sac; Fr, frontal air sac; Ju, junk; Ln, left naris; Ma, mandible; Mo, monkey/ phonic lips; MT, muscle/tendon layer; Rn, right naris; Ro, rostrum; So, spermaceti organ; $\mathrm{p} 0, \ldots, \mathrm{p} 4$, pulse components of a sperm whale click showing their relative level and constant interpulse interval (IPI).
Based on photogrammetric size estimation and matched sound recordings, Gordon (1991) derived an empirical relation between sperm whale length and IPI (Goold and Jones, 1995; Goold et al., 1996) using a sound speed in spermaceti oil of some $1350 \mathrm{~m} / \mathrm{sec}$ (Flewellen and Morris, 1978; Goold et al., 1996). Investigations on stranded animals (Møhl, 2001) and onboard recordings with acoustic tags (Madsen et al., 2002a) show a good agreement between length estimates and the predictions of the Gordon equation. Recently, Rhinelander and Dawson (2004) verified the correlation between IPIs and the size of the animal. However, they also reported some discrepancy between photogrammetric length estimates and length prediction from the Gordon equation, and proposed a slightly different equation for large animals. The contention is therefore that the IPI is indeed related to the size of the animal and that such information is conveyed in all sperm whale usual clicks.

Despite the potential for acoustic size estimation of sperm whale stocks, the technique has only been employed in a few studies (Adler-Fenchel, 1980; Leaper et al., 2002; Pavan et al., 2000; Drouot et al., 2004; Miller et al., 2004; Rhinelander and Dawson, 2004). While there may be a number of reasons for this, one likely relates to the fact that recorded sperm whale clicks seldom conform to the clear multipulse structure depicted in textbooks and papers. Clicks with complex wave forms and pulse structures that cannot be accounted for on the basis of a fixed IPI must often be excluded from the analysis (Gordon, 1987; 1991; Goold, 1996; Pavan et al., 1997; Drouot et al., 2004, Rendell and Whitehead, 2004; Rhinelander and Dawson, 2004;).

In an attempt to explore the mechanisms behind the complex structures of sperm whale usual clicks that do not conform to the clear multipulse pattern, we analyzed acoustic data from a field experiment combining a towed hydrophone array in the far field with an acoustic and orientation recording tag attached to a sperm whale. This approach provided recordings of both the emitted clicks in a fixed recording aspect (from the tag) and in varying, but known recording aspects in the far field (from the towed array) (Zimmer et al., 2005). We demonstrate that the interpulse structure of usual clicks from a sperm whale varies considerably and that sperm whale body length, therefore, is not estimated correctly from the IPI of clicks recorded off the body axis. The multipulse structure of sperm whale usual clicks can be related to the recording aspect of the whale by a more complex geometric model. We develop such a model, consistent with the bent horn hypothesis of sound production, and show that the observed variations in IPI with aspect closely follow those predicted by the model.

\section{MATERIALS AND METHODS}

The following analysis is based on sperm whale data recorded in the Ligurian Sea in 2001 during Sirena-01, a field trial organized by the NATO Undersea Research Centre (NURC) as part of its Marine Mammal Acoustic Risk Mitigation program. Data collected during Sirena trials included: visual observation of animals at the surface, passive sonar detection and tracking while animals were diving, and tagging of animals with a compact acoustic and orientation recorder, the DTAG, (Johnson and Tyack, 2003) developed at the Woods Hole Oceanographic Institution (WHOI).

Clicking sperm whales were detected by a passive sonar system developed at NURC consisting of a horizontal line array of 128 hydrophones, a real-time digital beamformer and sonar display system. The hydrophone array was towed just below any substantial thermocline at a depth of about $80 \mathrm{~m}$. The received sound was processed and visualized in real-time on a passive sonar display (Zimmer et al., 2003), and archived on a $240 \mathrm{MBit} / \mathrm{s}$ digital tape recorder, together with relevant nonacoustic data such as array depth and ship's position, heading, and speed. The hydrophones of the towed array were set to an effective saturation level of $140 \mathrm{~dB}_{\text {peak }}$ re: $1 \mu \mathrm{Pa}$ and sampled with 16 bit resolution at $31.25 \mathrm{kHz}$, allowing a maximum bandwidth of about $15 \mathrm{kHz}$.

The passive sonar was able to track sperm whales throughout their foraging dives, but not to record the detailed orientation or short-term movements of the animal between clicks. For this task a DTAG was attached to the whale, recording the sounds and movement patterns of the whale with high resolution (Johnson and Tyack, 2003). Key features of the DTAG in 2001 were 12-bit analog-to-digital conversion of a hydrophone signal, a sampling rate of $32 \mathrm{kHz}$ and a clipping level set to $153 \mathrm{~dB}_{\text {peak }} r e: 1 \mu \mathrm{Pa}$. The pressure sensor, three-axis accelerometers and three-axis magnetometers were all sampled at $47 \mathrm{~Hz}$ and strict synchrony was maintained between audio and sensor sampling.

The procedure for tagging sperm whales was based on the following scheme: once a sperm whale was located acoustically and visually, a small workboat was deployed from the NRV Alliance to attempt tagging. Responses to tagging were monitored visually and acoustically from NRV 


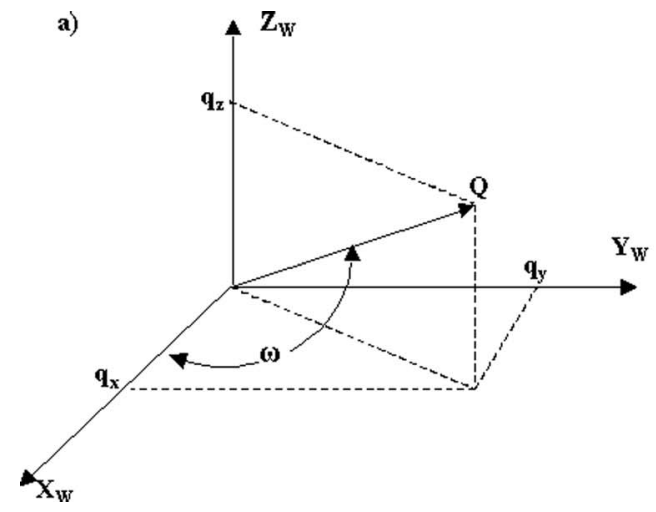

b)

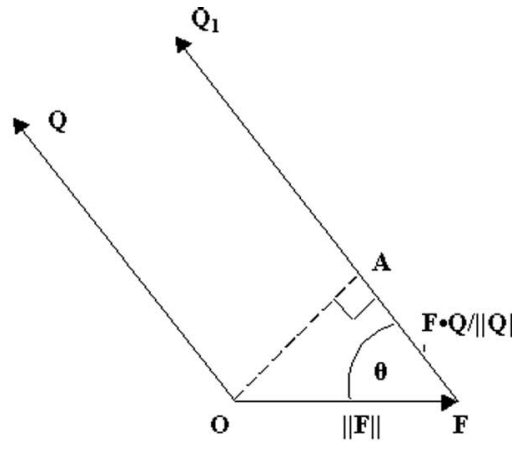

FIG. 2. (a) The general geometry of the whale coordinate system $\left(X_{W}, Y_{W}, Z_{W}\right)$, indicating the range vector $Q$ in whale coordinates $Q=\left(q_{x}, q_{y}, q_{z}\right)$, and the off-axis angle $\omega$. (b) The geometry used to estimate the path length difference between two acoustic rays, with $Q$ connecting the sound source directly to the receiver and $Q_{1}$ describing the sound path from a reflector at point $F$. F॰ $\hat{Q}_{1}$ is the dot product between vector $F$ and the unit vector $\hat{Q}_{1}$ and describes the projection of vector $F$ on vector $\hat{Q}_{1}$ and produces the distance between the points $F$ and $A$.

Alliance as well as from the small workboat. After tagging, the whale was followed visually when it surfaced close to the ship, acoustically when it was clicking at depth, and using a radio direction finder to track a VHF radio transmitter on the tag when the whale surfaced.

On 2-October-2001, a DTAG was attached for nearly $7 \mathrm{~h}$ to a whale (SW01_275b) estimated by visual observation (Miller et al., 2004) to be about $12 \mathrm{~m}$ long. While tagged, the whale performed eight complete deep foraging dives to depths of 550-900 m. Data analysis was performed in multiple steps described in detail in Zimmer et al. (2005), where the same data set was used to determine the beampattern of the usual sperm whale click. The underwater track of the whale was reconstructed from visual sightings, passive acoustics, and tag measurements of whale orientation and depth. Clicks made by the tagged whale were then detected in the DTAG recording and the corresponding clicks were identified in the recording from the towed hydrophone array.

The positions of source (whale) and receiver (towed array) were transformed from geo-referenced axes (i.e., east, north, zenith) to a whale-relative coordinate system (i.e., forward, left, up). The roll $(\alpha)$, pitch $(\beta)$, and heading $(\gamma)$ angles of the whale were estimated from the tag data during the reconstruction phase. Using these angles, the coordinate system of the whale is defined by the following vectors:

$$
\begin{aligned}
& X_{W}=T_{1}, \\
& Y_{W}=T_{2} \cos \alpha+T_{3} \sin \alpha, \\
& Z_{W}=T_{3} \cos \alpha-T_{2} \sin \alpha,
\end{aligned}
$$

where

$$
T_{1}=\left(\begin{array}{c}
\cos \beta \cos \gamma \\
\cos \beta \sin \gamma \\
\sin \beta
\end{array}\right), \quad T_{2}=\left(\begin{array}{c}
\sin \gamma \\
-\cos \gamma \\
0
\end{array}\right),
$$

$$
T_{3}=\left(\begin{array}{c}
-\sin \beta \cos \gamma \\
-\sin \beta \sin \gamma \\
\cos \beta
\end{array}\right),
$$

where $X_{W}$ points in the forward direction and defines the longitudinal axis, $Y_{W}$ points to left of the whale, and $Z_{W}$ points dorsally. For simplicity, we consider the whale coordinates to have their origin at the phonic lips.

The geo-referenced vector $R$ pointing from the whale to the receiver [Fig. 2(a)] can now be expressed in whale-frame coordinates by $Q=[q x, q y, q z]^{T}$ where

$$
\begin{aligned}
& q_{x}=X_{W} \circ R, \\
& q_{y}=Y_{W} \circ R, \\
& q_{z}=Z_{W} \circ R,
\end{aligned}
$$

and $X \circ R$ denotes the dot product between vector $X$ and $Y$. Note that while the two vectors $R$ and $Q$ have the same length, that is $\|R\|=\|Q\|$, they are expressed in different coordinate systems ( $R$ is geo-referenced and $Q$ is in whale-frame coordinates). The off-axis angle $\omega$ describes the angle between the forward direction of the whale (assumed to be the acoustic axis) and the direction to the receiver and is defined by the relation $\cos \omega=q_{x}$ [Fig. 2(a)].

The key geometric approximation used in the following pertains to the path length difference between a direct and reflected sound ray as perceived by a distant observer [Fig. 2(b)]. If the vectors between the source and observer, and between the reflector and observer are, respectively, $Q$ and $Q_{1}$, and $F$ is the vector connecting the source and reflector, then the path length difference, $\lambda$, is approximated for $\|Q\|$ $\gg\|F\|$ by

$$
\begin{aligned}
\lambda & =\|F\|+\left\|Q_{1}\right\|-\|Q\| \approx\|F\|+F \circ Q_{1} /\left\|Q_{1}\right\| \\
& =\|F\|+F \circ Q /\|Q\|=\|F\|(1+\cos \theta),
\end{aligned}
$$

where $\theta$ is the angle between $F$ and $Q_{1}$ (or, equivalently, $Q$ for a distant observer). The approximation is excellent for $k=\|Q\| /\|F\|>100$ giving a maximum path length error of about $\|F\| / 2 k$. 

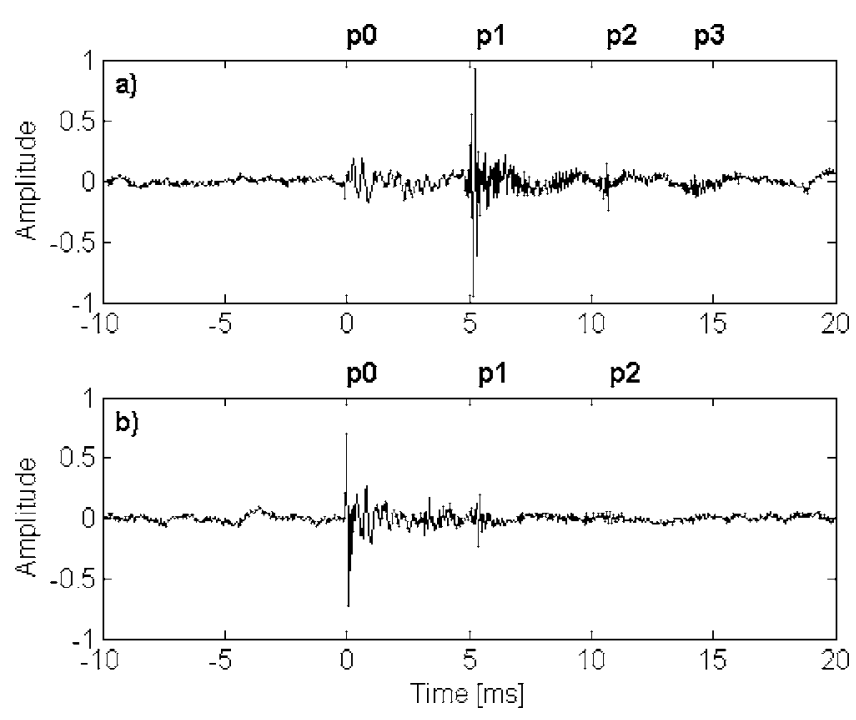

FIG. 3. Usual sperm whale clicks from forward, panel (a) and backward direction, panel (b), as measured by the remote receiver. The different component pulses in the clicks are denoted p0, p1, p2, and $\mathrm{p} 3$.

\section{RESULTS AND DISCUSSION}

The combination of an orientation recording tag on the whale and an acoustic recorder in the far field allowed us to derive the off-axis aspect of the recorder with respect to the whale for each emitted click (Zimmer et al., 2005). Figure 3(a) provides an example of a click recorded close to the forward direction [azimuth of $9^{\circ}$ and elevation of $7^{\circ}$ ( $\mathrm{Zim}$ mer et al., 2005)] and thereby close to the acoustic axis of the whale. The click structure conforms to the findings of Møhl et al. (2003) having a weak p0 pulse, a dominating p1 pulse followed by a weaker $\mathrm{p} 2$ pulse. The interpulse intervals can clearly be measured by either the time difference between $\mathrm{p} 0$ and $\mathrm{p} 1$ or $\mathrm{p} 1$ and $\mathrm{p} 2$. Figure 3(b) displays another click recorded close to the body axis, but at an off-axis angle of $160^{\circ}$ (Zimmer et al., 2005) and therefore from behind the animal. In this case the $\mathrm{p} 0$ pulse dominates because the recording aspect is now close to the acoustic axis of $\mathrm{p} 0$ and off the acoustic axis of the powerful p1 pulse (Madsen et al., 2002a; Zimmer et al., 2005). Note that the amplitudes are normalized in Figs. 3(a) and 3(b). In reality the on-axis source level of the p1 pulse is some $40 \mathrm{~dB}$ higher than that of the p0 pulse. It is evident from Fig. 3(b) that the interpulse intervals can be easily measured when recording directly behind the animal, e.g., in the footprint of a diving whale (Gordon, 1987; Goold and Jones, 1995; Rhinelander and Dawson, 2004). Thus, recordings made on the body axis of a sperm whale, whether in front or behind, will show a clear multipulse structure and consistent IPIs.

However, most sperm whale recordings are made with a single hydrophone and with the whale in an unknown orientation. When analyzing such recordings it is often painfully clear that most sperm whale clicks do not conform to the clean pattern outlined in Fig. 3, looking more like the click displayed in Fig. 4. In such complex wave forms, the click is still multipulsed, but the pulses are not evenly spaced and it is far from obvious how to number the various pulses according to the pattern of Fig. 1 with $\mathrm{p} 0$ to $\mathrm{pN}$. Such pulses

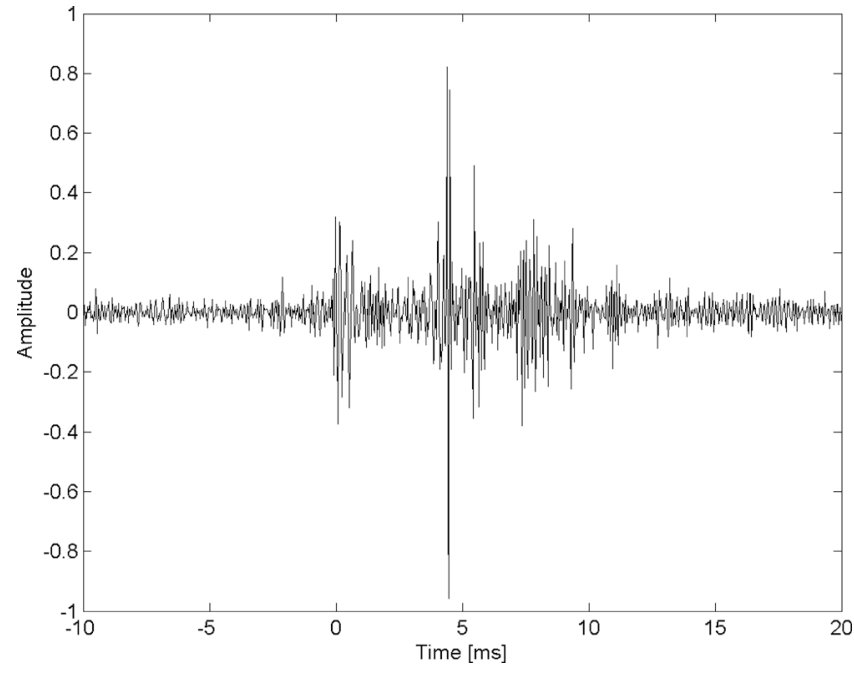

FIG. 4. Single off-axis sperm whale click as measured by the remote receiver. Time 0 corresponds to the reception of the $\mathrm{p} 0$ pulse.

are therefore often discarded from the process of acoustic size estimation (Gordon, 1991; Goold, 1996; Rendell and Whitehead, 2004) or used uncritically which in turn renders a range of varying and sometimes unrealistic size estimates for the same animal. It is challenging to make sense of the wave forms of such off-axis clicks even when the recording aspect is known but when a large number of consecutive clicks are aligned in a stacked plot a pattern emerges (Fig. 5). In this plot, nearly 290 consecutive clicks, recorded from a single hydrophone in the towed array, were stacked together by aligning the clicks at the onset of the $\mathrm{p} 0$ pulses. Later arriving pulses have varying interpulse delays between 2 and $6 \mathrm{~ms}$, and if such delays were used as IPI estimates for acoustic size determination (following the formulas of Gordon, 1991 or Rhinelander and Dawson, 2004), the estimated size of the whale would vary between 6 and $13 \mathrm{~m}$. It should be emphasized that this variation in the pulse structure gen-

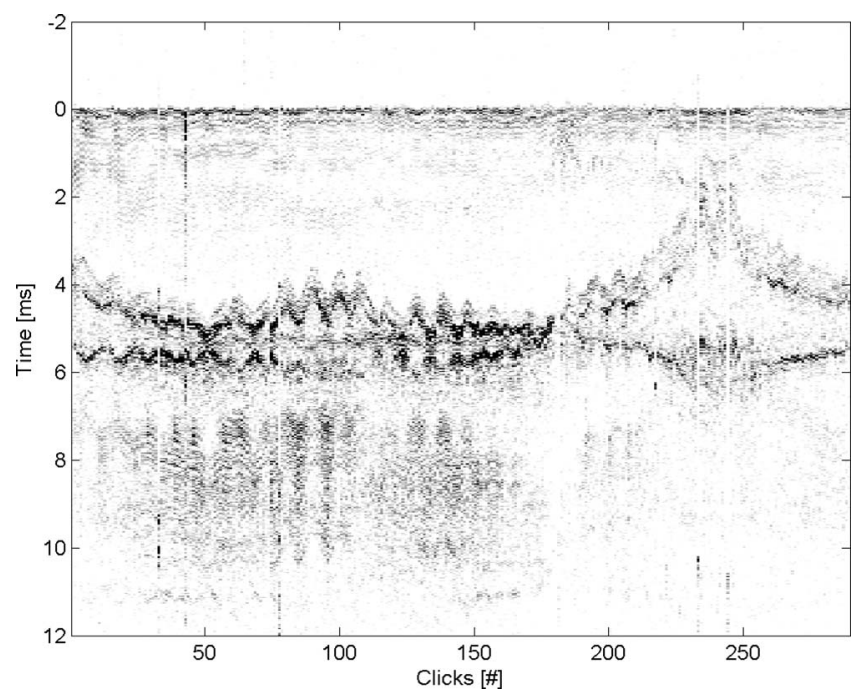

FIG. 5. Multiple sperm whale clicks, as recorded from a remote hydrophone, stacked horizontally. Each vertical slice contains the envelope of a received click aligned at the p0 pulse. The oscillating multipulse structure is visible at time delays of up to $6 \mathrm{~ms}$. 


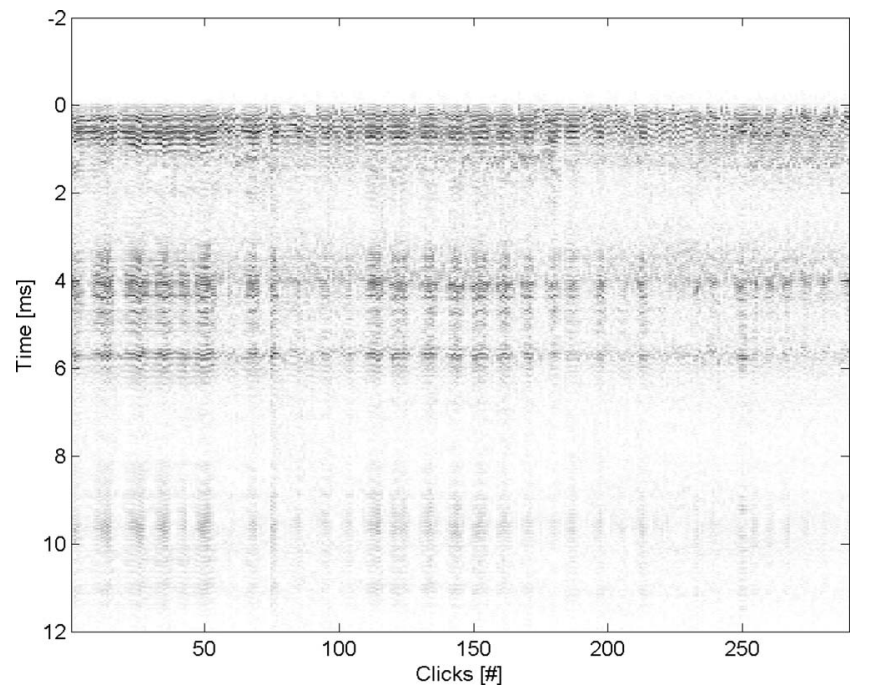

FIG. 6. Stackplot of the same consecutive sperm whale clicks shown in Fig. 5 , but measured by the DTAG that was attached to the clicking whale.

erally cannot be overcome by processing the received signal: both autocorrelation and cepstral analyses of isolated sperm whale clicks will yield incorrect IPIs.

Closer inspection of Fig. 5 reveals that the multipulse pattern develops over time and it seems that there is a correlation between the wave form of a click and the wave forms of the clicks preceding and following it. We hypothesize that such variability in the temporal structure can either (1) relate to variability in the sound production system or (2) be caused by changing geometry between the clicking whale and the receiver in the far field. To address the first hypothesis, we aligned the same clicks displayed in Fig. 4, but as recorded by the tag on the animal (Fig. 6). This approach ensures that the recording aspect is close to being fixed, and that changes in the received wave form most likely relate to changes in the output of the sound generator rather than aspect changes (Madsen et al., 2002a). Figure 6 shows that while the received levels vary slightly over the course of time, the multipulse structure is stable with pulses at around 0,4 , and $5.7 \mathrm{~ms}$. There are small fluctuations, which may relate to minor changes in the recording conformation between the tag at the dorsal fin and the sound generator in the front of the nasal complex of the swimming whale. However, compared to the large IPI fluctuations of the same clicks recorded in the far field, the pulse structure is stable, and it is safe to conclude that source modifications cannot explain the fluctuations in the multipulse structure observed in the far field (sensu Madsen et al., 2002a).

Given that the pulse structure of the emitted wave forms is stable over time intervals of minutes, we analyzed the data for aspect-dependency in the far field. To do so, we compared the observed signals to a simplified source model based on the bent horn theory. We know from tag (Madsen et al., 2002a), cadaver (Møhl et al., 2002), and far field recordings (Møhl et al., 2003; Zimmer et al., 2005) that the sperm whale sound generator can be viewed as a sound source with two reflectors and several discrete exit paths (Fig. 7). While the functional morphology and acoustic properties are likely more complicated, a simple compartmental model of this

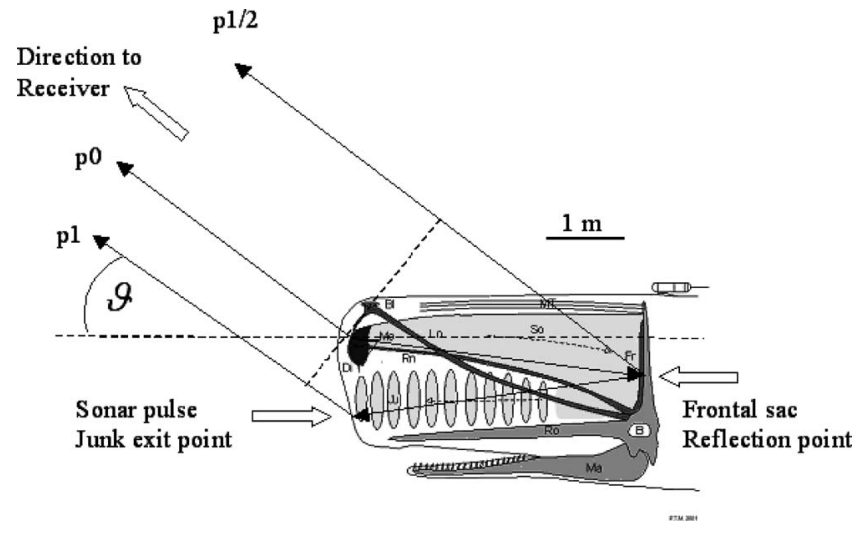

FIG. 7. Diagram of the modified bent-horn model of sound production in sperm whales; $\theta$, off-axis angle of the receiver with respect to the acoustic axis; 0 , primary pulse generated by the phonic lips; p1, highly directional sonar pulse; p1/2, pulse reflected from frontal air sac.

form provides a tractable framework in which to explore the relationship between observer aspect and pulse delays in the observed signals.

If there is no leakage from the system other than at the anterior surface of the nose, it is expected from the simple pipe experiment of Norris and Harvey (1972) that the interpulse intervals would be constant irrespective of the recording aspect. On the other hand, if the sound energy is not entirely contained in the spermaceti compartments, a reflection should result from the frontal air sac and result in a new pulse, that we coin p1/2 ("p-half"), in addition to the p0 and p1 pulses predicted by the Norris and Harvey (1972) and the bent-horn amendment (Møhl, 1978; Møhl, 2001). It is predicted that the p1/2 pulse will appear in the far field with an orientation-dependent delay relative to the $\mathrm{p} 0$ pulse varying between 0 (i.e., merging with the p0) when recorded behind the whale, and the two-way-travel time of the nose (i.e., merging with $\mathrm{p} 1$ ) when recorded directly in front of the whale on the acoustic axis of the p1 pulse.

To predict the $\mathrm{p} 1 / 2$ time delay as a function of aspect, we first need to define the position of the reflection point on the frontal sac. This organ is posterior to the spermaceti organ and below the longitudinal axis as defined earlier, and so has a position vector $F=\left[f_{x} 0 f_{z}\right]^{T}$ where $f_{x}$ is the longitudinal distance from the phonic lips to the frontal sac, essentially the length of the spermaceti organ. For simplicity, we model the frontal sac as a single reflection point ventral of the longitudinal axis $\left(f_{z}<0\right)$ and with no lateral offset $\left(f_{y}=0\right)$ with respect to the phonic lips, as justified by the lateral symmetry of the frontal sac (Madsen, 2002b).

The apparent time delay, $\tau_{1 / 2}$, of the $\mathrm{p} 1 / 2$ pulse, relative to $\mathrm{p} 0$, is the combination of two factors [sensu Fig. 2(b)]:

the travel time of sound from the phonic lips to frontal sac. This is given by $\|F\| / v_{s}$ where $v_{s}$ is the speed of sound in the spermaceti organ, and

(ii) the travel time difference between the path from frontal sac to observer as compared to the path from phonic lips to observer. For a distant observer (specifically, for $\|R\| \gg\|F\|)$, the aspect of every point of the whale's nose is essentially the same and so the 

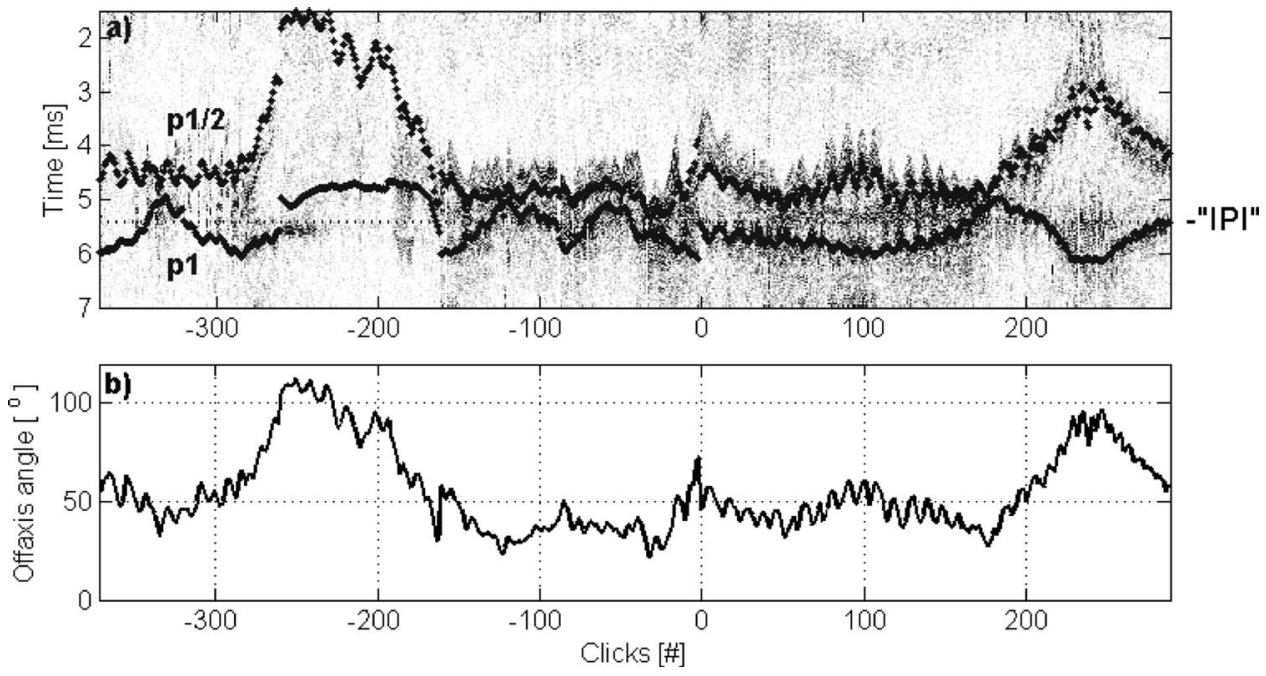

FIG. 8. (a) Multiple sperm whale clicks stacked and zoomed with model overlaid. The lower dotted line describes the modeled $\mathrm{p} 1$ pulse, the upper dotted line the modeled $\mathrm{p} 1 / 2$ pulse, and the horizontal dashed line corresponds to the nominal IPI. Clicks with positive click numbers correspond to the clicks shown also in Fig. 5 that were used for least-squares parameter estimation. Clicks with negative click numbers precede the clicks of Fig. 5, and are shown together with the predicted $\mathrm{p} 1$ and $\mathrm{p} 1 / 2$ values. (b) The estimated off-axis angle for each click.

path length difference is simply given by $\delta_{1 / 2}=F \circ \hat{Q}$, where $\hat{Q}=Q /\|Q\|$ is the unit vector in the direction of the observer (expressed in whale-frame coordinates). The path length difference $\delta_{1 / 2}$ is a function of aspect with $\delta_{1 / 2} \approx 0$ at broadside and $\delta_{1 / 2} \approx\|F\|$ or $-\|F\|$ when the aspect is $0^{\circ}$ or $180^{\circ}$, respectively.

Converting path length difference to travel time is complicated by the fact that the segment of path may pass predominantly through connective tissue and water (aspects close to broadside), through the spermaceti organ (aspects close to $0^{\circ}$ ), or through the whale body (aspects larger than $90^{\circ}$ ). The speed of sound differs in these different tissues, but by using the sound speed in the spermaceti organ to convert path length to time delay, we make the least error for aspects close to 0 . Combining these results, the apparent time delay of the p1/2 pulse can be expressed as: $\tau_{1 / 2}=(\|F\|+F \circ \hat{Q}) / v_{s}$.

The results of the $\tau_{1 / 2}$ prediction for the click subset of Fig. 5 are shown by the top dotted line in Fig. 8(a) where the reflector positions were determined so as to minimize the square error between the predicted and measured delays giving $f_{x}=3.6 \mathrm{~m}$ and $f_{z}=-0.6 \mathrm{~m}$. It can be seen that the predicted p1/2 delays fit the actual delays quite well, explaining the intermediate pulses in off-axis clicks such as that of Fig. 3. As demonstrated in Fig. 8(a), the predicted arrival time of the $\mathrm{p} 1 / 2$ pulse consistently matches a pulse component in the off-axis clicks supporting the p1/2 pulse hypothesis. The model is successful at predicting the arrival time of this pulse in clicks that were not used to estimate the model parameters (the negatively numbered clicks in Fig. 8) based only on the off-axis angle, an important validity test for the model. However, it is also evident in Fig. 8 that the p1/2 pulse arrives over a fairly broad spread of delays rather than a single delay as predicted by the model indicating that the reflection from the frontal sac cannot be localized to a single point, as might be expected given the size of this reflector of about $1 \mathrm{~m}$ (Madsen, 2002b). The presence of a p1/2 pulse is nevertheless supported by the data and we conclude that a small part of the sound energy reflecting off the frontal sac must escape from the spermaceti compartments and radiate directly into the water, giving rise to the $1 / 2$ pulse with an aspectdependent delay between the $\mathrm{p} 0$ and the $\mathrm{p} 1$ pulse.

In the bent-horn model and its modifications (Møhl et al., 2003; Zimmer et al., 2005), the majority of the sound energy propagating backwards from the phonic lips is reflected at the frontal sac and directed into the junk complex to produce the highly directional and powerful p1 sonar pulse. It is assumed in the models that this pulse exits from the flat anterior surface of the junk (Møhl, 2001) although this has not been demonstrated experimentally on a live animal. If the model is correct, then the time delay, $\tau_{1}$, between the $\mathrm{p} 0$ and the $\mathrm{p} 1$ pulse as observed by a remote listener will be aspect dependent due to the vertical separation of the two exit points. In particular, $\tau_{1}$ ought to vary with changes in pitch and roll. To test this hypothesis, we first define the location vector of a presumed radiation point from the junk to be $J=\left[00 j_{z}\right]^{T}$, where it is assumed that the junk is located ventral to the phonic lips by $j_{z}$ and that any lateral and longitudinal displacement is small in comparison. As with the p1/2 pulse, we assemble the p0-p1 time delay out of the following parameters:

(i) the travel time of sound from phonic lips to frontal sac, $\|F\| / v_{s}$.

(ii) the travel time of sound from frontal sac to anterior surface of the junk, $\|J-F\| / v_{s}$ where we use $v_{s}$ as a proxy for the speed of sound in the junk.

(iii) the travel time difference between the path from junk radiator to observer ( $\mathrm{p} 1$ ) as compared to the path from phonic lips to observer (p0). Again making the assumption of a distant observer, the path length difference is $\delta_{1}=J \circ \hat{Q}$, where $\hat{Q}$ is redefined as the unit vector from the junk radiator to the observer. $\delta_{1}$ is close to zero when the observer is in the horizontal plane of the whale and is $\pm j_{z}$ when the observer is dorsal or ventral of the phonic lips, respectively.

For off-axis angles less than $90^{\circ}$, both the $\mathrm{p} 0$ and the $\mathrm{p} 1$ pulse travel mainly in water, while for off-axis angles greater than $90^{\circ}$, both pulses travel through whale tissue with variable sound speed. To simplify the presentation, we use the 
speed of sound in water $v_{w}$, which was measured to be about $1510 \mathrm{~m} / \mathrm{s}$ to the whale depth (Zimmer et al., 2003). Combining these results, the apparent time delay of the 1 pulse is: $\tau_{1}=(\|F\|+\|J-F\|) / v_{s}+J \circ \hat{Q} / v_{w}$.

The prediction of $\tau_{1}$ using this model is superimposed on the multipulse patterns in Fig. 8(a) where, as before, the position parameter is determined by least-squares error fitting. It is evident (lower dotted line) that the modeled p1 delay is a good fit to the actual p1 delay both in clicks used for the parameter estimation (the positive click numbers in Fig. 8), and also for novel clicks (negative click numbers in Fig. 8). The temporal offset between the phonic lips and the junk surface that gave the best fit between the model and data was $0.73 \mathrm{~ms}$. Using a sound speed in water of $1510 \mathrm{~m} / \mathrm{s}$, this delay corresponds to a junk exit point centered at $110 \mathrm{~cm}$ ventral of the phonic lips, which for a $12 \mathrm{~m}$ sperm whale is close to the center of the flat anterior junk surface (Fig. 7; Madsen, 2002b).

We have shown that observed far-field signals from an instrumented sperm whale are consistent with there being three, spatially separated exit paths for sound from the nasal complex. A portion of the energy in the initial p0 pulse, generated by the phonic lips, radiates directly into the water but most of the caudally directed part of the sound energy passes through the spermaceti to be reflected at the frontal sac producing a second weak sound source, dubbed here $\mathrm{p} 1 / 2$. The main part of the forward reflected energy from the frontal sac passes through the junk to form the p1 pulse, which is the primary sonar pulse for echolocation (Madsen et al., 2002b; Møhl et al., 2003). Results given here match the predictions of the bent horn model and are the first direct evidence from a live sperm whale that the powerful, and highly directional $\mathrm{p} 1$ pulse is emitted via the anterior surface of the junk into the water, a result that supports the amendment to the original Norris and Harvey model (1972) proposed by Møhl (1978; 2001). The demonstration that the junk is the exit for sonar pulses in sperm whales also supports evolutionary scenarios of the functional morphology in this species in which the junk is seen as being homologous to the sound conducting melon of smaller toothed whales (Schenkan and Purves, 1972; Cranford et al., 1996). The junk of the sperm whale nose is therefore most likely both anatomically and functionally homologous to the melon of smaller toothed whales.

While the basic geometric predictions of the bent horn model have been confirmed by the data (Fig. 8), it should be noted that some components of the off-axis clicks are still unaccounted for. The energy arriving after the $\mathrm{p} 1$ pulse and seen at delays between 7 and $10 \mathrm{~ms}$ in clicks 20 through 150 in Fig. 5 has not been explained and we are unable to say unequivocally whether this is due to reflections within the whale or reflections from objects in the water column. However, they are most likely generated in the nose since reflections from the sea surface or bottom can be excluded as the delays are too short being equivalent to source-reflector separations of $10-15 \mathrm{~m}$.

The empirical validation of the geometry of the bent horn model for sound propagation in the sperm whale nose may prove helpful in analyzing signals from animals of un- known orientation. An understanding of the basic geometry behind the complex pulse structure opens the possibility that animal orientation and size could be derived acoustically from off-axis clicks if a large enough sample set is analyzed as in Fig. 8. However, until a proven algorithm for this is presented, we strongly recommend that IPIs used for acoustic size estimation are derived from clicks recorded right behind a diving whale (Gordon, 1991; Goold, 1996; Rhinelander and Dawson, 2004) or close to the acoustic axis of the p1 pulse in front of the whale (Møhl et al., 2003).

We have shown that the time delays $\tau_{1 / 2}$ and $\tau_{1}$ of pulses in sperm whale usual clicks, when observed from a remote position, depend on the relative orientation of the whale. We expect that fluking is the main cause of the short-period oscillation in off-axis angle seen in Fig. 8(b) and this oscillation is consistent with delay variations in the $\mathrm{p} 1 / 2$ and $\mathrm{p} 1$ pulses visible in Fig. 8(a). Amplitude variations of similar scale are also evident in the clicks recorded by the tag (Fig. 6 ) indicating that these are related to relative motion of the tag and sound source, consistent with body flexure during fluking. The indication is that the propulsion mechanism of the whale modulates the orientation of the sound generation system and consequently the acoustic transmission axis of the biosonar. As the beamwidth of the p1 pulse has been reported to be very narrow [directivity index of $27 \mathrm{~dB}$ or a $-3 \mathrm{~dB}$ beam width of about $8.3^{\circ}$, Møhl et al., 2003, Zimmer et al., (2005)], it would seem that motion-induced variation of the acoustic axis should impact sonar functionality. Our results suggest that sperm whales do not completely stabilize the direction of the 1 pulse during fluking, or perhaps intentionally use the motion-induced variation to insonify larger volumes.

The multipulse structure of sperm whale clicks has been proposed to serve the purpose of conveying information about size to conspecifics and one of the hypotheses behind the hypertrophy of the nasal complex relates to sexual selection on that basis (Cranford, 1999). Provided that the auditory system of sperm whales can cope with the problems of forward masking and the differentiation of very small time delays (Madsen, 2002a), sperm whales could tell the size of a nearby clicking whale from its usual clicks if the IPIs were stable and independent of aspect. The observed variation of IPIs with aspect will clearly complicate such size estimation. However, the predictable nature of the multipulse structure as a function of aspect angle demonstrated here, may, along with spectral cues (Møhl et al., 2003), provide information to nearby whales regarding the orientation of the clicking whale similar to the situation for calls of delphinids (Miller, 2002; Lammers and Au, 2003). The low decay rate of coda clicks compared to usual clicks (Madsen et al., 2002a) holds more potential for IPI decoding, but it remains to be seen how the wave forms of coda clicks appear in different recording aspects (Rendell and Whitehead, 2004). If the production of coda clicks does not involve the junk complex as suggested by Madsen et al.(2002a), then sound energy would principally exit from the nose at the distal and frontal sacs leading to a highly aspect-dependent variation in the multipulse structure. This is in apparent contrast to observations in the field of stable coda IPIs (Rendell and Whitehead, 2004). Fu- 
ture studies should investigate this discrepancy experimentally and address the biomechanics of different sound production modes in the sperm whale nose.

\section{CONCLUSION}

Remote recordings of sperm whale clicks together with simultaneous acoustic and orientation information measured by a tag on the same whale have been combined to investigate the origin of the multipulse structure in sperm whale usual clicks. These recordings were compared to a lumpedparameter geometric model for sound reflection in the sperm whale nose based on the bent-horn model developed by Møhl and co-workers (Møhl, 1978; 2001; Møhl et al., 2003) and on recently reported three-dimensional radiation patterns of $\mathrm{p} 0$ and $\mathrm{p} 1$ pulses of sperm whale usual clicks (Zimmer et al., 2005).

One consequence of the bent-horn model should be a small variation in time delay between the $\mathrm{p} 0$ and $\mathrm{p} 1$ pulse as a function of aspect angle due to the spatial separation between the phonic lips, where the p0 pulse is generated, and the center of the flat anterior junk surface, where the $\mathrm{p} 1$ pulse is hypothesized to exit. A careful analysis of off-axis measurements confirms the predicted variations and allows us to conclude that the powerful p1 sonar pulse is indeed emitted from the junk surface. Production of usual sonar clicks in sperm whales therefore involves both the spermaceti organ and the junk complex, and the latter is accordingly both anatomically and functionally homologous to the melon of smaller toothed whales.

The existence of a wide backward-oriented beam of the p0 pulse demonstrates that small amounts of sound energy leak from the spermaceti organ when the p0 pulse travels from the phonic lips to the frontal sac (Zimmer et al., 2005). Similar leakage is also likely for sound that is reflected from the frontal sac back into the junk complex and spermaceti organ. We validate this prediction by the empirical demonstration of a so far undescribed intermediate pulse, called $\mathrm{p} 1 / 2$, which is characterized by a large and aspect-dependent variation in time delay relative to the p0 pulse. Fitting the observed p1/2 pulse delays to a geometric model provides a prediction for the effective reflection point which is consistent with the center of the frontal sac. A consequence of the aspect-dependent pulse structure described here is that both the size and aspect of a clicking whale are effectively coded in the pulse intervals and this may allow conspecifics to establish the size and orientation of other clicking whales. However, this coding represents a confound for field methods that estimate the size of clicking whales based on the interpulse-interval of single clicks and such estimates should be confined to clicks measured directly in front (on-axis) or directly caudal to minimize error in the apparent IPI due to the aspect-dependent $\mathrm{p} 1 / 2$ pulse.

\section{ACKNOWLEDGMENTS}

This work was carried out within the Acoustic Risk Mitigation Program of NATO Undersea Research Centre. Thanks to the science party and ship's crew on the research cruise during which these data were collected. A. Shorter, T.
Hurst, and A. Bocconcelli (WHOI) provided skilled engineering support. We thank B. Møhl and M. Wahlberg for helpful discussions and constructive critique on previous versions of the manuscript. The whale was tagged under a scientific research permit No. 981-1578-00 issued by the US National Marine Fisheries Service to Peter L. Tyack. This is Contribution No. 11361 from the Woods Hole Oceanographic Institution. The Woods Hole Oceanographic Institution Animal Care Use Committee approved this research. This work was funded by grants to from the Office of Naval Research Grant Nos. N00014-99-1-0819 and No. N0001401-1-0705, and the Packard Foundation.

Adler-Fenchel, H. S. (1980). "Acoustically derived estimate of the size distribution for a sample of sperm whales (Physeter catodon) in the Western North Atlantic," Can. J. Fish. Aquat. Sci. 37, 2358-2361.

Backus, R. H., and Schevill, W. E. (1966). "Physeter Clicks," in Whales, Dolphins and Porpoises, edited by K. S. Norris (University of California Press, Berkeley), pp. 510-527.

Cranford, T. W. (1999). "The sperm whale's nose: Sexual selection on a grand scale?," Marine Mammal Sci. 15, 1133-1157.

Cranford, T. W., Amundin, M., and Norris, K. S. (1996). "Functional morphology and homology in the odontocete nasal complex: Implications for sound generation," J. Morphol. 228, 223-285.

Drouot, V., Gannier, A., and Goold, J. C. (2004). "Diving and feeding behavior of sperm whales (Physeter macrocephalus) in the northwestern Mediterranean Sea," Aquatic Mammals 30, 419-426.

Flewellen, C. G., and Morris, R. J. (1978). "Sound velocity measurements on samples from the spermaceti organ of the sperm whale (Physeter catodon)," Deep-Sea Res. 25, 269-277.

Goold, J. C. (1996). "Signal processing techniques for acoustic measurement of sperm whale body lengths," J. Acoust. Soc. Am. 100, 3431-3441.

Goold, J. C., Bennell, J. D., and Jones, S. E. (1996). "Sound velocity measurements in spermaceti oil under the combined influences of temperature and pressure," Deep-Sea Res., Part I 43, 961-969.

Goold, J. C., and Jones, S. E. (1995). "Time and frequency domain characteristics of sperm whale clicks," J. Acoust. Soc. Am. 98, 1279-1291.

Gordon, J. C. D. (1987). "The behaviour and ecology of sperm whales off Sri Lanka," Ph.D. thesis, Darwin College, Cambridge.

Gordon, J. C. D. (1991). "Evaluation of a method for determining the length of sperm whales (Physeter catodon) from their vocalizations," J. Zool. Lond. 224, 301-314.

Johnson, M., and Tyack, P. L. (2003). "A digital acoustic recording tag for measuring the response of wild marine mammals to sound," IEEE J. Ocean. Eng. 28, 3-12.

Lammers, M. O., and Au, W. W. L. (2003). "Directionality in the whistles of Hawaiian spinner dolphins (Stenella longirostris): A signal feature to cue direction of movement?," Marine Mammal Sci. 19, 249-264.

Leaper, R., Chappell, O., and Gordon, J. C. D. (2002). "The development of practical techniques for surveying sperm whale populations acoustically," Rep. Int. Whal. Comm. 42, 549-560.

Madsen, P. T. (2002a). "Sperm whale sound production-in the acoustic realm of the biggest nose on record," in Sperm whale sound production, Ph.D. dissertation, University of Aarhus, Denmark.

Madsen, P. T. (2002b). "Morphology of the sperm whale head: A review and some new findings," in Sperm whale sound production, Ph.D. dissertation, University of Aarhus, Denmark.

Madsen, P. T., Carder, D. A., Au, W. W., Nachtigall, P. E., Mohl, B., and Ridgway, S. H. (2003). "Sound production in neonate sperm whales," J. Acoust. Soc. Am. 113, 2988-2991.

Madsen, P. T., Payne, R., Kristiansen, N. U., Wahlberg, M., Kerr, I., and Møhl, B. (2002a). "Sperm whale sound production studied with ultrasound time/depth—recording tags," J. Exp. Biol. 205, 1899-1906.

Madsen, P. T., Wahlberg, M., and Møhl, B. (2002b). "Male sperm whale (Physeter macrocephalus) acoustics in a high latitude habitat: Implications for echolocation and communication," Behav. Ecol. Sociobiol. 53, 31-41.

Miller, P. J. O. (2002). "Mixed-directionality of killer whale stereotyped calls: A direction of movement cue?," Behav. Ecol. Sociobiol. 52, 262270.

Miller, P. J. O., Johnson, M. P., Tyack, P. L., and Terray, E. A. (2004).

"Swimming gaits, passive drag and buoyancy of diving sperm whales 
Physeter macrocephalus," J. Exp. Biol. 207, 1953-1967.

Møhl, B. (1978). "Kaskelot Klik. Akustiske egenskaber, dannelsesmåde, og en mulig funktion," in Liv og Lyd (in danish), edited by A. Michelsen and S. G. Dalsgaard (University of Odense Press, Odense, Denmark), pp. 201204.

Møhl, B. (2001). "Sound transmission in the nose of the sperm whale Physeter catodon. A post mortem study," J. Comp. Physiol. [A] 187, 335-340.

Møhl, B., Madsen, P. T., Wahlberg, M., Au, W. W. L., Nachtigall, P. E., and Ridgway, S. (2002). "Sound transmission in the spermaceti complex of a recently expired sperm whale calf," ARLO 4, 19-24.

Møhl, B., Wahlberg, M., Madsen, P. T., Heerfordt, A., and Lund, A. (2003). "The monopulsed nature of sperm whale clicks," J. Acoust. Soc. Am. 114, $1143-1154$.

Møhl, B., Wahlberg, M., Madsen, P. T., Miller, L. A., and Surlykke, A. (2000). "Sperm whale clicks: Directionality and source level revisited," J. Acoust. Soc. Am. 107, 638-648.

Nishiwaki, N., Oshumi, S., and Maeda, Y. (1963). "Changes in form of the sperm whale accompanied with growth," Sci. Rep. Wh. Res. Inst. Tokyo 17, 1-13.

Norris, K. S., and Harvey, G. W. (1972). "A theory for the function of the spermaceti organ of the sperm whale (Physter catodon L.)," in Animal Orientation and Navigation, edited by S. R. Galler, K. Schmidt-Koenig, G. J. Jacobs, and R. E. Belleville, SP-262 (NASA, Washington, DC), pp. 397-417.
Pavan, G., Hayward, T. J., Borsani, J. F., Priano, M., Manghi, M., Fossati, C., and Gordon, J. C. D. (2000). "Time patterns of sperm whale codas recorded in the Mediterranean Sea 1985-1996," J. Acoust. Soc. Am. 107, 3487-3495.

Pavan, G., Priano, M., Manghi, M., and Fossati, C. (1997). "Software tools for real-time IPI measurements on sperm whale sounds," Proc. Institute Acoust. 19, 157-164.

Rendell, L., and Whitehead, H. (2004). "Do sperm whales share coda vocalizations? Insights into coda usage from acoustic size measurements," Anim. Behav. 67, 865-874.

Rhinelander, M. Q., and Dawson, S. M. (2004). "Measuring sperm whales from their clicks: Stability of interpulse intervals and validation that they indicate whale length," J. Acoust. Soc. Am. 115, 1826-1831.

Schenkan, E. J., and Purves, P. E. (1972). "The comparative anatomy of the nasal tract and the function of the spermaceti organ in the Physeteridae (Mammalia, Odontoceti)," Bidragen tot de Dierkunde 43, 93-112.

Zimmer, W. M. X., Johnson, M. P., D’Amico, A., and Tyack, P. L. (2003). "Combining data from a multisensor tag and passive sonar to determine the diving behavior of a sperm whale (Physeter macrocephalus," IEEE J. Ocean. Eng. 28, 13-28.

Zimmer, W. M. X., Tyack, P. L., Johnson, M. P., and Madsen, P. T. (2005). "Three-dimensional beam pattern of regular sperm whale clicks confirms bent-horn hypothesis," J. Acoust. Soc. Am. 117, 1473-1485. 